\title{
Partial pharyngolaryngectomy with infrahyoid flap: Our experience
}

\author{
Sophie Cortese ${ }^{\mathrm{a}, *}$, Enrico Muratori ${ }^{\mathrm{a}, 1}$, Romina Mastronicola ${ }^{\mathrm{a}, \mathrm{c}}$, Medarine Roch $^{\mathrm{a}}$, Emilie Beulque ${ }^{\mathrm{a}}$, \\ P. Rauch ${ }^{\mathrm{a}}$, Lucie Dekerle ${ }^{\mathrm{a}}$, Alberto Deganello ${ }^{\mathrm{b}}$, Gilles Dolivet ${ }^{\mathrm{a}, \mathrm{c}}$ \\ ${ }^{a}$ Institut de Cancérologie de Lorraine, Department of Surgical Oncology, 6 avenue de Bourgogne, 54519 Vandoeuvre-lès-Nancy, France \\ ${ }^{\mathrm{b}}$ Department of Otorhinolaryngology-Head and Neck Surgery, University of Brescia, Brescia, Italy \\ ' CNRS, CRAN, F-54000, France
}

\section{A R T I C L E I N F O}

\section{Keywords:}

Infrahyoid flap

Hypopharyngeal squamous cell carcinoma

\begin{abstract}
A B S T R A C T
Aim: We evaluated a cohort of advanced hypopharyngeal squamous cell carcinoma, treated with conservative surgery, reconstruction with infrahyoid flap and radio-chemotherapy.

Methods: We used partial pharyngo-laryngectomy and radio-chemotherapy to treat fifty-seven patients with stage III-IV hypopharyngeal SCC from November 1994 to December 2011. Clinical examination and speech therapy evaluation were used for estimation of laryngeal function.

Results: All patients received a partial pharyngo-laryngectomy. All patients underwent neck dissection; 56 patients received bilateral neck dissection. Reconstruction was achieved by infra-hyoid flap. Five-year overall and disease-specific survival rates were $54.4 \%$ and $61.4 \%$, respectively. Successful laryngeal function preservation with complete five-year remission was achieved in $44 \%$ of the patients.

Conclusion: Selected even if advanced carcinomas of the hypopharynx maybe treated with partial pharyngolaryngectomy with reconstruction with pedicled flap. Both oncological and functional results showed a good outcome.
\end{abstract}

\section{Introduction}

Squamous cell carcinoma of the hypopharynx (HPC) is the least common of the upper aerodigestive cancers, representing $5 \%$ of headand-neck cancers [1]. Because of factors such as advanced disease, patient comorbidity, and a high incidence of distant metastases, patients with HPC have the worst prognosis of all head-and-neck cancer patients [1-4]. Hypopharyngeal cancer is most common in men in their mid-60s and with a lower socioeconomic status. It is caused by alcohol or smoking (or both). Many studies reported a low proportion $(0 \%-11 \%)$ of p16 HPV-positive tumors in patients with HPC $[5,6]$. Treatment has varied over time and between jurisdictions, but essentially, until the late 1990s, the typical treatments were radiotherapy, surgery, or a combination of the two, with no evidence of the superiority of one treatment modality for all cases [7]. With the advent, based on randomized trials and meta-analyses of concomitant chemoradiotherapy (CCRT) for head-and-neck squamous cell carcinomas [8-11]. As oncologists have moved more toward the concept of organ preservation, other treatments have included induction chemoradiotherapy $[3,12,13]$ and transoral laser surgery $[14,15]$. A complete review of treatment options published by Takes et al. [16] concluded that more evidence was needed to determine optimal treatment and that "treatments should be individualized by knowledgeable multidisciplinary teams". Definitive radiation therapy is generally considered an effective therapeutic approach for T1 and selected T2 hypopharyngeal carcinoma especially in presence of comorbidities [17]. In advanced stage diseases must be considered a more extensive treatment such as surgery and CCRT [18]. Hypopharyngeal reconstruction has been a major challenge over the years. Every partial laryngectomy aiming to maintain laryngeal functions must guarantee adequate airway together with at least one functioning crico-arytenoid unit, in horizontal partial laryngeal surgery these requirements are achieved with an end to end reconstruction of the airway by means of a pexy, but in case of hypopharyngeal-laryngeal resection the resulting defect is more complex and the transposition of a flap is usually required. In literature several techniques deal with the reconstruction after total laryngectomy with circumferential or partial hypopharyngeal resection, but very few reports describe the reconstruction of pharyngo-laryngeal defects resulting from HPC resection and laryngeal preservation surgery. Many surgical techniques has been developed and purposed [19]

\footnotetext{
* Corresponding author.

E-mail addresses: Cortese.sophie@gmail.com, s.cortese@nancy.unicancer.fr (S. Cortese).

${ }^{1}$ Otorhinolaryngology Unit, Department of Surgery, Arcispedale Santa Maria Nuova, Center for Clinical abd Basic Research, Reggio Emilia, Italy.
} 
ranging from deltopectoral flap [20] to pectoralis major flap [21-23] to gastric pull up. With the more recent rise of microvascular and free flap reconstruction both surgical approaches and functional results in hypopharyngeal cancer, for "patch" or circumferential reconstruction, have changed have improved. Microvascular flaps commonly used are jejunum, radial forearm free flap, or anterolateral thigh flap [24]. We report our series of HPC treated with laryngeal conservation surgery where the reconstruction was effectively provided by the infrahyoid flap. This flap has proven its utility in various head and neck sites and it presents several characteristics that make it very suitable for pharyngolaryngeal reconstruction.

\section{Patients and methods}

\subsection{Patients}

Criteria for patient selection for this study had included: (1) squamous cell carcinoma of the hypopharynx, (2) stages III and IV status, (3) absence of infiltration of the apex of the pyriform sinus or retro-cricoid and parapharyngeal regions and (4) reconstruction obtained by means of infrahyoid flap. From November 1996 to December 2014, 57 patients have been admitted for hypopharyngeal cancer and received formal or extended partial supraglottic laryngo-pharyngectomy for hypopharyngeal squamous cell carcinoma. To obtain the results, the following have been taken into account after re-evaluation of patients chart: history of disease, clinical, radiological and pathologic characteristics of lesions, post-operative complications, radiation therapy modalities, delay for retrieval of tracheotomy and nasogastric feeding tube, complications and duration of local control. The local extension of the tumor has been evaluated preoperative by means of systematical panendoscopies. In each case, pathologic examination revealed squamous cell carcinoma. During the panendoscopy, an endoscopic percutaneous gastrostomy has been eventually placed in patients who presented dysphagia or with a previewed post-operative eating rehabilitation longer than 3 weeks. Only one patient had been already treated and presented with a post-radiotherapy relapse. Three patients among the 57 initially included died within a few weeks following the operation and were, therefore, not taken into account for functional and locoregional control assessment. All of the patients included in the present study were staged by the TNM staging system as recommended by the 2010 American Joint Committee on Cancer on clinical staging.

\subsection{Surgical technique}

All patients were operated on using basically two surgical procedures. The first one was the supraglottic pharyngo-laryngectomy technique described by Ogura [25]; it is suitable for tumors limited to the medial wall of the pyriform sinus with possible extension to the aryepiglottic fold. In case of lateral wall of the pyriform sinus or to posterior hypopharyngeal wall involvement, the same technique had been used, adding an extended pharyngectomy perfected by Hamoir [26]. In case of hemilaryngeal fixation, a second technique was used as described by Urken and consisting of partial pharyngo-laryngectomy, including the hemicricoid and hemithyroid cartilages [27]. Both techniques speculate an ipsilateral thyroid gland lobectomy. Neck dissection was bilateral in all cases. Primary closure was achieved with a local infrahyoid muscle flap in all patients.

Infrahyoid flap is a myocutaneous flap pedicled on the superior thyroid vessels. Described for the first time by Wang in 1979, technical improvements have been introduced by Dolivet et al. [28] especially to enhance the venous drainage and aesthetic results at the donor site.

The infrahyoid muscles included sternohyoid, thyrohyoid, sternothyroid and omohyoid constitute the anatomical substratum of the flap, completed by the plathysma and the overlying skin.

A fusiform skin paddle located at the same side of the pharyngeal resection and centred over infrahyoid muscles and the cricoid region is

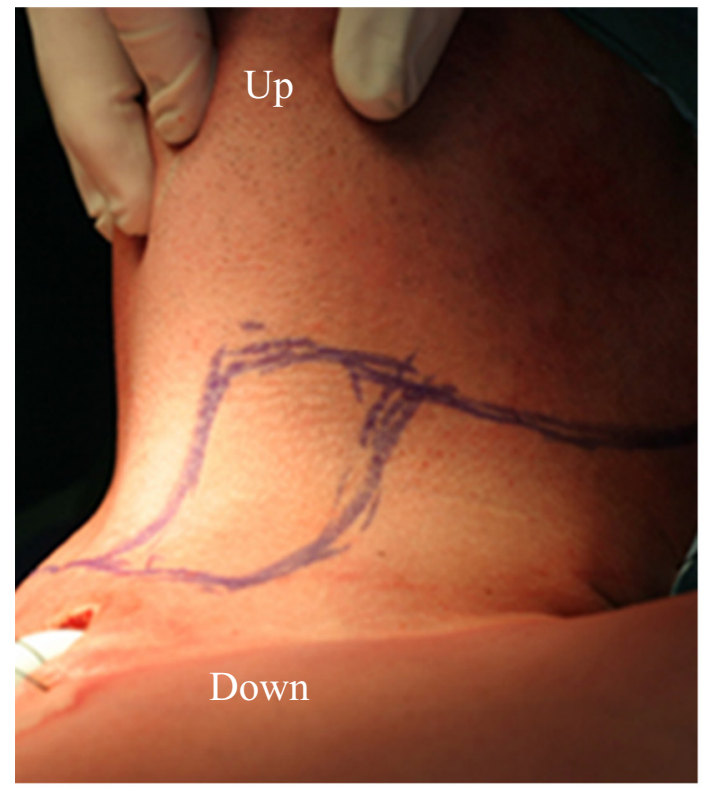

a

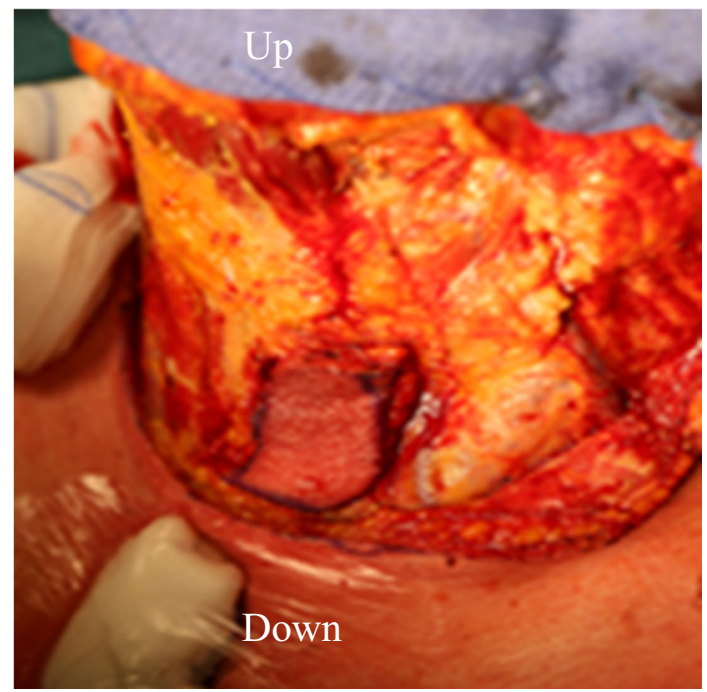

b

Fig. 1. Left infrahyoid flap.

a: Drawing of the limits of flap before incision on the same side of the tumor. The skin paddle is included in the incision of neck dissection.

b: The skin and platysma are incised all around the skin paddle to allow prompt choke perforator vessels opening.

outlined and is included in neck incision (Fig. 1a). The medial limit of the flap lies at the midline, the upper limit at the level of hyoid bone, and the lower limit at the suprasternal notch. This flap can measure up to $10 \mathrm{~cm}$ in its greatest length and reach a distance of $15 \mathrm{~cm}$ around its rotation axis. The flap harvesting is fast and the donor site can be primary closed to the original operating field when the skin paddle is not $>5 \mathrm{~cm}$.

First, skin and platysma are incised all around the skin paddle to allow prompt choke perforator vessels opening (Fig. 1b). Infrahyoid flap harvest is normally performed after the ipsilateral neck dissection and before tumor resection (Fig. 2a). The technique in harvesting of the flap is common to those of other districts. The surgical anatomy and the harvesting of the infra hyoid flap are well described in precedent publications [29-31]. The flap is raised from lateral to medial and from caudal to cephal. 


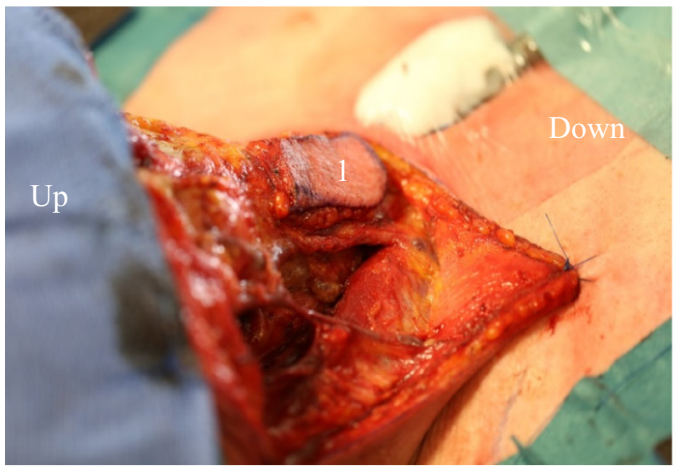

a

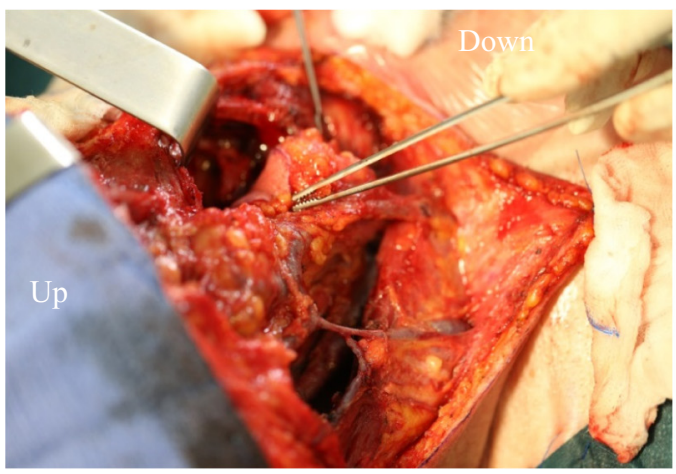

c

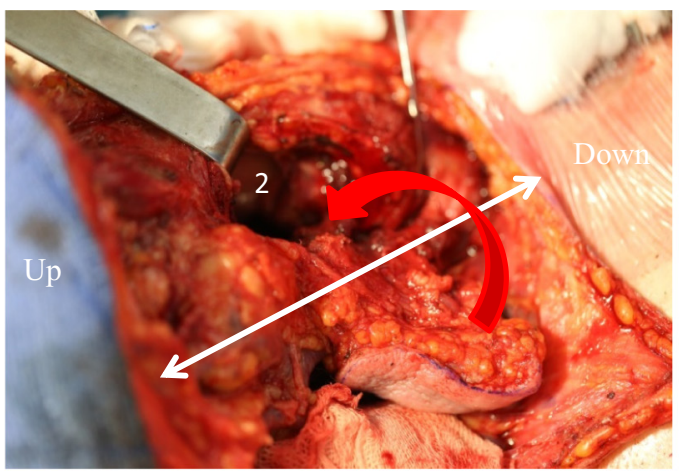

b

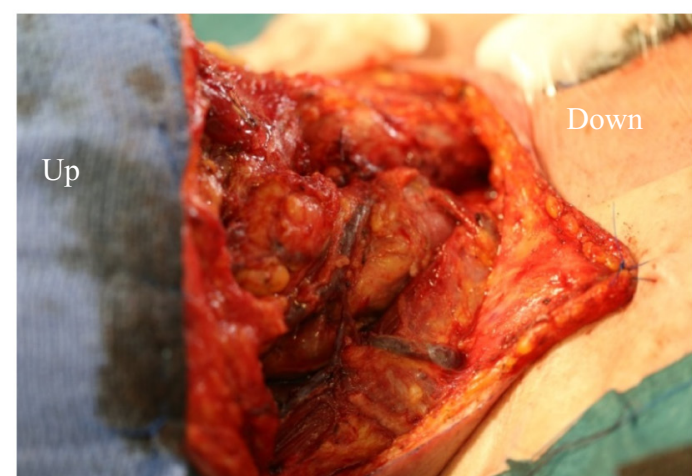

d

Fig. 2. Surgical procedure.

a: The infrahyoid flap [1] is elevated after neck dissection.

c, d: After tumor resection, the defect [2] is filled by performing a rotation of the flap toward the inside. Double layer sutures are held frontward.

The elevation starts by sectioning the sternohyoid and sternothyroid muscles from the suprasternal notch. Then the flap is elevated over the capsule of the thyroid lobe. The cricothyroid artery and vein, the posterior branch of the superior thyroid artery and vein after being ligated and cut are harvested with the flap. Medially, the linea alba is sectioned. The infrahyoid muscles are released from the thyroid cartilage plan and the hyoid bone. The hyoid insertions of the sternohyoid and omohyoid muscles are sectioned to allow the mobility of the flap and the exposure of the pharyngolarynx. Once tumor resection completed, the reconstruction is obtained by turning the flap medially (Fig. 2b, c). The skin paddle is sutured with reabsorbable to the remaining mucosa. Double layer sutures are held frontward (Fig. 2d). Flap harvesting may occur in communication with the tracheotomy and contamination to the wound bed. To avoid this, the thyroid isthmus is simply sutured to the subcutaneous tissue.

\subsection{Adjuvant therapy}

According to $\mathrm{pN}$ staging (positive lymph nodes, extracapsular spread) and prognostic factors such as borderline or positive margins, vascular and perineural involvement, post-operative adjuvant therapies were administer to patients. Normally, patients were treated with parallel-opposed lateral fields for the upper neck, including the primary site and an anterior field for the lower neck. Total dose administered ranged from 60 to $64 \mathrm{~Gy}$. Patients received daily $2 \mathrm{~Gy}$ fractions for 6-6.5 weeks. Chemotherapy has not been given in older patients ( $>80$ years) and/or patients with severe comorbidities.

\subsection{Post-operative evaluation}

If the removal of tracheotomy cannula and/or feeding support devices (nasogastric or gastrostomy tube) was possible, it was annotated.
As well as laryngeal preservation (ability to speak, no tracheotomy and deglutition without symptomatic aspiration) was the functional aim; a 1 -year evaluation in survived patients has been done with a special focus on vocal function, swallowing difficulties and social life.

\subsection{Statistical methods}

All data were analyzed using Stata/SE 12.0 (StataCorp., College Station, TX). Patient survival curves were estimated using the Kaplan-Meier and product-limit methods. Overall survival analysis was based on death from any cause. For the calculation of cause-specific survival, patients were censored if death was not directly related to the pyriform sinus cancer. Survival interval was measured from the date of surgery in the department to last consultation, phone inquiry or death. Comparisons between survival curves were made using the log-rank test with a significance level based on a $p$-value of $<0.05 .1$.

\section{Results}

\subsection{Patient population and treatment}

In the study, fifty-seven patients were analyzed. All patients referred with a squamous cell carcinoma of the hypopharynx. Baseline characteristics are showed in Table 1.

All lesions had advanced stages; among them, twenty three patients had a clinical stage III, the remaining thirty four had a stage IV tumor. Clinical TNM evaluation is summarized in Table 2. As described before, all patients received a partial pharyngo-laryngectomy. Forty-one patients received a partial pharyngo-laryngectomy according to Ogura/ Hamoir technique, 12 patients received an Urken's hemilaryngealpharyngectomy. The remaining 4 patients received a more tailored surgery eventually extended to base of the tongue or lateral pharyngeal 
Table 1

Baseline patients characteristics.

\begin{tabular}{ll}
\hline Age (years, median \pm SD) & $58.5 \pm 9.1$ \\
Male & $56(98.2 \%)$ \\
Female & $1(1.8 \%)$ \\
Stage & \\
III & $23(40.4 \%)$ \\
IV & $34(59.6 \%)$ \\
Site & \\
Hypopharynx & $24(42.1 \%)$ \\
Hypopharynx-larynx & $30(52.6 \%)$ \\
Hypopharynx-oropharynx & $3(5.3 \%)$ \\
Synchronic primary tumor & $3(5.3 \%)$ \\
\hline
\end{tabular}

Table 2

Clinical TNM evaluation.

\begin{tabular}{llllll}
\hline & T1 & T2 & T3 & T4 & Total \\
\hline N 0 & 0 & 0 & 3 & 1 & 4 \\
N 1 & 1 & 15 & 4 & 0 & 20 \\
N 2a & 0 & 0 & 4 & 0 & 4 \\
N 2b & 0 & 9 & 6 & 1 & 16 \\
N 2c & 1 & 4 & 3 & 3 & 11 \\
N 3 & 0 & 2 & 0 & 0 & 2 \\
Total & 2 & 30 & 20 & 5 & 57 \\
\hline
\end{tabular}

wall. All patients underwent bilateral modified radical neck dissection type III. Four patients received a one-side radical neck dissection. Median operative time had been $4 \mathrm{~h} 15 \mathrm{~min} \pm 43 \mathrm{~min}$ (range $3 \mathrm{~h}-6 \mathrm{~h}$ ). One patient had been referred for salvage treatment of recurrence after poor response to radiation therapy, and was considered suitable for conservation surgery. Reconstruction required in all cases a local infrahyoid muscle flap. One patient received also a pectoral myo-cutaneous flap to cover a defect bigger than previewed. Fifty-four patients received post-operative radiation therapy. Thirty patients received post-operative chemotherapy.

Pathological examination reveals a confirmation of clinical stage in 44 patients (77.2\%) whilst 11 patients got an upstaged lesion (19.3\%) and $2(3.5 \%)$ a downstaged one. Pathologic examination of the resected tumor revealed disease free margins in 46 patients $(80.7 \%)$, and microscopically positive or border-line in eleven cases on the initial resection margins. In these cases, margins were enlarged until histologically disease free on frozen section. However, four patients had frozen section negative margins revealed positive in the definitive evaluation. Nodal metastases were histopathologically diagnosed in 52 patients (91.2\%), among which 15 (28.8\%) had bilateral involvement and 32 $(61.5 \%)$ were found to have extracapsular spread.

\subsection{Complications}

One patient died in the immediate post-operative course for unknown reason while sleeping (possible acute asphyxia due to a blood plug). Twenty-four patients presented local complications: 5 patients were affected by pneumonia; 2 hemorrhages had occurred (one needed a surgical revision); 7 patients experienced deep wound infection, one of those evolved in a salivary fistula. The overall incidence of pharyngocutaneous fistula has been 5 out of 57 patients (9\%), all successfully treated with compressive dressing. Five patients needed blood transfusion. None flap necrosis has been seen.

\subsection{Functional results}

Tracheotomy tube was removed with a median delay of 18 postoperative days (range: 7-40 days). To define the median, the patients who underwent adjuvant therapy with a previewed risk for treatment have been excluded.

Five patients $(9 \%)$ could not remove their tracheotomy at 1-year

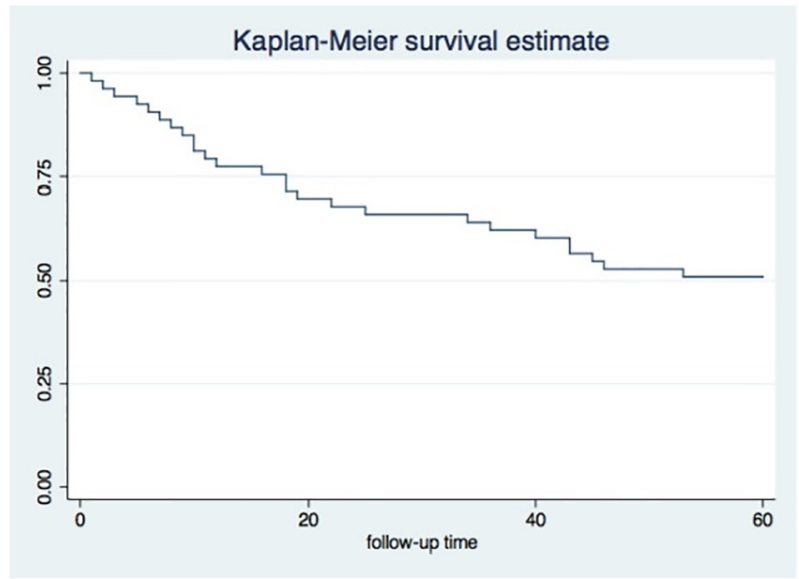

a

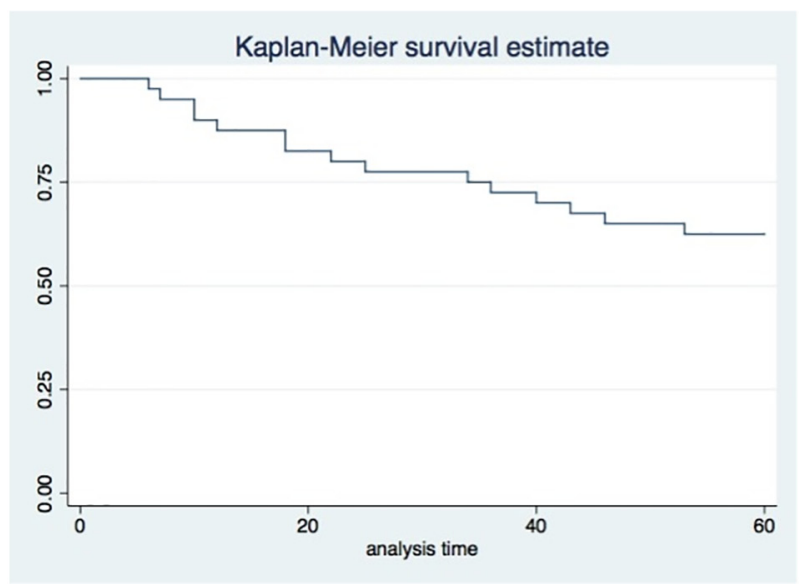

$\mathrm{b}$

Fig. 3. Survival and loco-regional control.

a: Overall 5-years survival.

b: Disease free survival.

evaluation. Laryngeal preservation (ability to speak, no tracheotomy and deglutition without symptomatic aspiration) was achieved in 42 of 55 functionally assessed cases (76\%) at the end of the treatment. Deglutition was assessed clinically and radiologically with modified barium swallow. Overall, in $75 \%$ of the patients, deglutition, with the ability to tolerate liquid and solid food was obtained.

\subsection{Survival and loco-regional control}

To assess the survival rate four patients have been removed, as they were lost in follow-up before the fifth year. The overall 5-years survival rate was $50.9 \%$ (Fig. 3a).

Fifteen patients $(28.3 \%)$ died because of cancer evolution, among them $11(20.8 \%)$ had distant metastasis and $6(11.3 \%)$ had a loco-regional recurrence. $3(5.7 \%)$ patients died because of a second primary tumor; $8(15.1 \%)$ patients died for others causes such as 2 intestinal infarction, 6 pulmonary complications). The disease free survival observed in our series was $61.4 \%$ (Fig. 3b).

\section{Discussion}

The hypopharyngeal carcinoma remains a major issue in head and neck cancer mainly because of late diagnosis and poor prognosis. 
Nowadays, total pharyngolaryngectomy (TPL) with adjuvant radiation therapy remains the most widely used treatment in cases of high-staged disease. However, TPL is encumbered by two main post-operative morbidities: permanent tracheostomy with loss of voice and the deglutition impairment. Moreover, whatever the therapeutic modality is used, overall 5-year survival rates, as reported in the literature, do not exceed 50\% [32-34]. During the last fifty years, the treatment paradigm for advanced hypopharyngeal cancer tends to shift from TPL to partial surgical or endoscopically performed approaches. The partial pharyngolaryngectomies (PLPs) were born as less traumatic minimally invasive procedures and have offered the opportunity of preserving function of the larynx in selected cases of hypopharyngeal cancer. In this respect, PLPs resections must encompass the tumor with adequate margins, must ensure airway patency, and must preserve at least one functioning crico-arytenoid unit. However, only selected cases will be suitable for this approach but the efficacy linked with tumor control of rising nonsurgical treatments, makes them a solution to obtain an improved quality of life [16] The first PLP reports advocated an immediate closure only with remaining pharyngeal mucosa and eventually a skin graft $[25,35]$. If this may be feasible with small defects, it is not acceptable for bigger mucosal resections in order to avoid pharyngo-cutaneous fistulas. To improve the functional results in the last decades a rising importance has been given to reconstruction; several techniques have been described including pectoralis major flap (PMF), gastric pull-up, free jejunal transposition, and fascio-cutaneous free flaps. An important role in establishing the most appropriate type of reconstruction is played by patient's comorbidities and specific risk factors [36-38]. Pedicled flaps, mainly the PMF, have been the first described for pharyngeal reconstructions, nowadays they remain the best choice as an alternative to free flaps in patients with general or local contraindications for microsurgery [17,36,38]. Nonetheless, a recent study compared pharyngeal reconstructions with PMF and free flaps showed no statistically significant differences in oral re-alimentation and decanulation time between the two groups; lower rate of medical and pulmonary complications in PMF but an increase of surgical ones. An explanation of these results may be the loss of suture tightness with subsequent onset of pharyngo-cutaneous fistulas and pharyngo-esophageal strictures due to PMF excessive thickness and weight. Concerning reconstructive procedures requiring a surgical weakening of digestive tract; major reconstructive procedures requiring laparotomy or laparoscopy may deplete their functional reserve and further impact their general status. In particular, free jejunal transposition is frequently associated with postoperative ileus, potentially leading to major complications, prolonged recovery, and longer hospital stay. Nouraei et al. recently evaluated 1589 pharyngo-laryngectomies suggesting that post-treatment complications occur frequently and increase short- and long-term mortality. Choice of reconstruction, and specifically the use of alimentary tract conduits, worsens short- and long-term survival. This may be due to added physiological stress the patient is placed under because of the opening of abdominal or thoracic and abdominal cavities [39]. Functional results are, in the best-case scenario, comparable with those obtained using fasciocutaneous free flaps. Currently, fasciocutaneous free flaps, in particular radial forearm (RF) and anterolateral thigh $[24,38,40]$ are considered among the main options for such a reconstructive purpose. Piazza et al. showed that first-line application of RF and ALT free flaps with long-lasting salivary by-pass stent in reconstruction after partial or total pharyngo-laryngectomy allows obtaining reduced incidences of both fistula and stenosis [24]. Nouraei et al. [39] supported the use of free flaps even in terms of overall survival regardless of tumor stage $(p<0.05)$. The treatment sequence should be taken in account; our believe, confirmed by literature, is that primary definitive radiotherapy followed by salvage surgery when indicated, is inferior in terms of survival and functional outcome [41]. Nonetheless, treatment with radiotherapy alone is reported to have a worse prognosis compared with combined treatment with surgery and radiotherapy, particularly in stage IV $[2,42,43]$. The only patient of the series treated after radiation failure died after 18 months because of a local relapse, moreover both decanulation and gastrostomy tube removal had been impossible to achieve before the fatality. This result follows the literature impression, even if statistical significance has not been obtained, this results is probably due to the flap impairment related to post-radiotherapy vessel depletion. Numerous open techniques initially described for laryngeal tumors were extended to encompass the resection of HPC, however traditional horizontal partial techniques often remove wide portions of healthy laryngeal structures not to get a resection free margin but to allow the reconstruction through a pexis. Our surgical approach is centered on obtaining an oncologically sound resection around the tumor rather that performing a standard predetermined partial pharyngo-laryngectomy, the resulting defect is reconstructed with the transposition of an infrahyoid flap. This myocutaneous pedicled flap, is thin and pliable flap and can usually provide a skin island of about $7 \times 4 \mathrm{~cm}$ from the central part of the anterior neck; in our series the skin paddle was usually smaller (about $6 \times 3 \mathrm{~cm}$ ) and then further tailored on defect dimensions. In our cohort our surgical approach demonstrated to be effective, ensuring timely healing and adequate functional results. In fact $91 \%$ of patients benefitted of the tracheostomy closure. Among patients with no respiratory risks, the cannula has been removed after $18 \pm 8.63$ days, and $75 \%$ of the patients returned to oral feeding. Takes et al. compared the overall survival and disease free survival of twenty retrospective studies conducted with the different techniques [24] paralleling the oncological results, we can spot that our series, with $54.4 \%$ as 5-year overall survival and $61.4 \% 5$ year disease-free interval is comparable to the endoscopic transoral series (Table 3).

We estimate that a reconstruction with a flap whether is pedicled or free permits a faster healing in order to begin as soon as possible the adjuvant therapies. In fact, compared a primary closure, the risk of pharyngeal fistula is reduced and related complications are lower. In addition, the functional results are better. Flap allows to restore the anatomy and to avoid retraction and stricture of pharynx. The most popular method for the management of defects in the head and neck area is represented by free flaps. However, in elderly patients or with comorbidities this reconstructive method is not adapted with a high risk of post-operative complications increase in the duration of stay and finally delay to adjuvant treatment. The infra-hyoid flap is proposed here as an alternative to free flaps. The simply and fast harvesting of the procedure represents a real advantage. In precedent publication [44]

Table 3

Partial pharyngo-laryngectomy series survival rates.

\begin{tabular}{llll}
\hline Treatment & $\begin{array}{l}\text { No. of } \\
\text { patients }\end{array}$ & $\begin{array}{l}\text { Overall survival } \\
\text { 5 year (4 year) } \\
\text { [3 year] -2 year- }\end{array}$ & $\begin{array}{l}\text { Disease-specific } \\
\text { survival } \\
5 \text { year (4 year) } \\
\text { [3 year] }\end{array}$ \\
& & & \\
& & & - \\
Open procedures & 85 & {$[59 \%$ ] } & $56 \%$ \\
Ogura et al. & 34 (T2) & - & - \\
Lacourreye et al. & 48 (T1/T2) & $47 \%$ & \\
Chevalier et al. & & T1: 78\% & \\
& & T2: 38\% & - \\
Makeieff et al. & 87 (T1/T2) & $60 \%$ & $65 \%$ \\
Plouin-Gaudon et al. & 34 & $50 \%$ & $95 \%$ (stage I/II) \\
Steiner et al. & 129 & $71 \%$ (stage I/II) & $69 \%$ (stage III/IV) \\
& & $47 \%$ (stage III/IV) & $58 \%$ \\
Rudert et al. & 29 & $48 \%$ & $(59 \%)$ \\
Vilaseca et al. & 28 & $(43 \%)$ & - \\
Kutter et al. & 58 & $-78 \%$ - & $96 \%$ (stage I/II) \\
Martin et al. & 172 & $68 \%$ (stage I/II) & $86 \%$ (stage III) \\
& & $64 \%$ (stage III) & $57 \%$ (stage IV) \\
& & $41 \%$ (stage IV) & $61 \%$ \\
Dolivet et al. & 57 (stage III/ & $54 \%$ & \\
& IV) & &
\end{tabular}


there was no significant difference in terms of postoperative complications, functionality and prognosis between pedicled flap and free flap for head and neck reconstruction. However, the infrahyoid flap offers a reduced thickness and bulkiness compared to PMF and this is probably the explanation in avoiding high rate of pharyngo-cutaneous fistula and pharyngo-esophageal strictures. On the other hand, the major contraindication in using the infrahyoid flap is due to its limited surface if compared to PMF or free flaps especially in covering defects reaching the cervical esophagus. Another point is the impossibility of using the flap as a backup in already surgically treated neck and an increased risk of failure in previous CCRT that compromises the quality of neck tissues. The limits of this flap are: the dimensional limitations, in our and most other series the average dimensions of the flap is $7 \times 4 \mathrm{~cm}$, previous thyroid surgery or neck dissection, and N3 neck metastasis. We consider that previous radiotherapy is not an absolute contraindication if the appearance of the cervical skin is normal, without fibrosis or teleangiectasias. Otherwise, the infrahyoid flap must be always be planned in advance.

\section{Conclusion}

Our series presented oncological and functional results comparable to the others wide series described in literature. The infrahyoid flap is a useful surgical instrument in hypopharyngeal reconstruction after partial pharyngo-laryngectomies and it should be considered, especially in case of general or local contraindications for microsurgery, as a smaller and lighter alternative to pectoralis major flap.

\section{References}

[1] Hall SF, Groome PA, Irish J, O'Sullivan B. The natural history of patients with squamous cell carcinoma of the hypopharynx. Laryngoscope 2008;118(8):1362-71.

[2] Sewnaik A, Hoorweg JJ, Knegt PP, Wieringa MH, van der Beek JM, Kerrebijn JD. Treatment of hypopharyngeal carcinoma: analysis of nationwide study in the Netherlands over a 10-year period. Clinical Otolaryngology 2005;30(1):52-7.

[3] Lefebvre JL, Rolland F, Tesselaar M, Bardet E, Leemans CR, Geoffrois L, et al. Phase 3 randomized trial on larynx preservation comparing sequential vs alternating chemotherapy and radiotherapy. J Natl Cancer Inst 2009;101(3):142-52.

[4] Hoffman HT, Karnell LH, Shah JP, Ariyan S, Brown GS, Fee WE, et al. Hypopharyngeal cancer patient care evaluation. Laryngoscope 1997;107(8):1005-17.

[5] Joo YH, Lee YS, Cho KJ, Park JO, Nam IC, Kim CS, et al. Characteristics and prognostic implications of high-risk HPV-associated hypopharyngeal cancers. Plos One 2013;8(11):e78718.

[6] Lewis Jr JS, Ukpo OC, Ma XJ, Flanagan JJ, Luo Y, Thorstad WL, et al. Transcriptionally-active high-risk human papillomavirus is rare in oral cavity and laryngeal/hypopharyngeal squamous cell carcinomas-a tissue microarray study utilizing E6/E7 mRNA in situ hybridization. Histopathology 2012;60(6):982-91.

[7] Hall SF, Groome PA, Irish J, O'Sullivan B. Radiotherapy or surgery for head and neck squamous cell cancer: establishing the baseline for hypopharyngeal carcinoma? Cancer 2009;115(24):5711-22.

[8] Pignon JP, Bourhis J, Domenge C, Designe L. Chemotherapy added to locoregional treatment for head and neck squamous-cell carcinoma: three meta-analyses of updated individual data. MACH-NC Collaborative Group. Meta-analysis of chemotherapy on head and neck cancer. Lancet 2000;355(9208):949-55.

[9] Pignon JP, le Maitre A, Maillard E, Bourhis J, Group M-NC. Meta-analysis of chemotherapy in head and neck cancer (MACH-NC): an update on 93 randomised trials and 17,346 patients. Radiother. Oncol. 2009;92(1):4-14.

[10] Blanchard P, Baujat B, Holostenco V, Bourredjem A, Baey C, Bourhis J, et al. Metaanalysis of chemotherapy in head and neck cancer (MACH-NC): a comprehensive analysis by tumour site. Radiother. Oncol. 2011;100(1):33-40.

[11] Hao D, Ritter MA, Oliver T, Browman GP. Platinum-based concurrent chemoradiotherapy for tumors of the head and neck and the esophagus. Semin Radiat Oncol 2006;16(1):10-9.

[12] Forastiere AA, Goepfert H, Maor M, Pajak TF, Weber R, Morrison W, et al. Concurrent chemotherapy and radiotherapy for organ preservation in advanced laryngeal cancer. N Engl J Med 2003;349(22):2091-8.

[13] Prades JM, Lallemant B, Garrel R, Reyt E, Righini C, Schmitt T, et al. Randomized phase III trial comparing induction chemotherapy followed by radiotherapy to concomitant chemoradiotherapy for laryngeal preservation in T3M0 pyriform sinus carcinoma. Acta Otolaryngol 2010;130(1):150-5.

[14] Martin A, Jackel MC, Christiansen H, Mahmoodzada M, Kron M, Steiner W. Organ preserving transoral laser microsurgery for cancer of the hypopharynx. Laryngoscope 2008;118(3):398-402.

[15] Lee TL, Wang LW, Mu-Hsin Chang P, Chu PY. Quality of life for patients with hypopharyngeal cancer after different therapeutic modalities. Head Neck
2013;35(2):280-5.

[16] Takes RP, Strojan P, Silver CE, Bradley PJ, Haigentz Jr M, Wolf GT, et al. Current trends in initial management of hypopharyngeal cancer: the declining use of open surgery. Head Neck 2012;34(2):270-81.

[17] Homma A, Sakashita T, Oridate N, Suzuki F, Suzuki S, Hatakeyama H, et al. Importance of comorbidity in hypopharyngeal cancer. Head Neck 2010;32(2):148-53.

[18] Bernier J, Cooper JS, Pajak TF, van Glabbeke M, Bourhis J, Forastiere A, et al Defining risk levels in locally advanced head and neck cancers: a comparative analysis of concurrent postoperative radiation plus chemotherapy trials of the EORTC (\#22931) and RTOG (\# 9501). Head Neck 2005;27(10):843-50.

[19] Richmon JD, Samji HA, Deschler DG. National laryngopharyngectomy and reconstructive surgery survey. Laryngoscope 2009;119(8):1472-8.

[20] Gilas T, Sako K, Razack MS, Bakamjian VY, Shedd DP, Calamel PM. Major head and neck reconstruction using the deltopectoral flap. A 20 year experience. Am J Surg 1986;152(4):430-4.

[21] Cusumano RJ, Silver CE, Brauer RJ, Strauch B. Pectoralis myocutaneous flap for replacement of cervical esophagus. Head Neck 1989;11(5):450-6.

[22] Lam KH, Wei WI, Lau WF. Avoiding stenosis in the tubed greater pectoral flap in pharyngeal repair. Archives of Otolaryngology-Head \& Neck Surgery 1987;113(4):428-31.

[23] Hirano M, Kurita S, Yoshida T, Tanaka H, Tai Y. Partial laryngopharyngectomy for piriform sinus carcinoma. Technique and preliminary results. Auris Nasus Larynx 1988;15(2):129-36.

[24] Piazza C, Bon FD, Paderno A, Grammatica A, Montalto N, Taglietti V, et al. Fasciocutaneous free flaps for reconstruction of hypopharyngeal defects Laryngoscope 2017;127(12):2731-7.

[25] Ogura JH, Marks JE, Freeman RB. Results of conservation surgery for cancers of the supraglottis and pyriform sinus. Laryngoscope 1980;90(4):591-600.

[26] Hamoir M, Lengele B, Rombaux P, El-Din AB, El Fouly P. Stretched radial forearm flap for reconstruction of the laryngopharynx: an alternative conservation procedure for radiation-failure carcinoma of the pyriform sinus. Laryngoscope 1999;109(8):1339-43.

[27] Urken ML, Blackwell K, Biller HF. Reconstruction of the laryngopharynx after hemicricoid/hemithyroid cartilage resection. Preliminary functional results. Archives of Otolaryngology-Head \& Neck Surgery 1997;123(11):1213-22.

[28] Dolivet G, Gangloff P, Sarini J, Ton Van J, Garron X, Guillemin F, et al. Modification of the infra hyoid musculo-cutaneous flap. European Journal of Surgical Oncology 2005;31(3):294-8.

[29] Deganello A, Leemans CR. The infrahyoid flap: a comprehensive review of an often overlooked reconstructive method. Oral Oncol 2014;50(8):704-10.

[30] Deganello A, Manciocco V, Dolivet G, Leemans CR, Spriano G. Infrahyoid fasciomyocutaneous flap as an alternative to free radial forearm flap in head and neck reconstruction. Head Neck 2007;29(3):285-91.

[31] Mirghani H, Meyer G, Hans S, Dolivet G, Perie S, Brasnu D, et al. The musculocutaneous infrahyoid flap: surgical key points. European Archives of Oto-RhinoLaryngology 2012;269(4):1213-7.

[32] Kraus DH, Zelefsky MJ, Brock HAJ, Huo J, Harrison LB, Shah JP. Combined surgery and radiation therapy for squamous cell carcinoma of the hypopharynx. Otolaryngology-Head and Neck Surgery 1997;116(6):637-41.

[33] Shah JP, Shaha AR, Spiro RH, Strong EW. Carcinoma of the hypopharynx. Am J Surg 1976;132(4):439-43.

[34] Kramer S, Gelber RD, Snow JB, Marcial VA, Lowry LD, Davis LW, et al. Combined radiation therapy and surgery in the management of advanced head and neck cancer: final report of study 73-03 of the Radiation Therapy Oncology Group. Head Neck Surg 1987;10(1):19-30.

[35] Ogura JH, Jurema AA, Watson RK. Partial laryngopharyngectomy and neck dissection for pyriform sinus cancer. Conservation surgery with immediate reconstruction. Laryngoscope 1960;70:1399-417.

[36] Deganello A, Gitti G, Parrinello G, Larotonda G, Meccariello G, Leemans CR, et al. Infrahyoid flap reconstruction of oral cavity and oropharyngeal defects in elderly patients with severe general comorbidities. Head Neck 2012;34(9):1299-305.

[37] Jubbal KT, Zavlin D, Suliman A. The effect of age on microsurgical free flap outcomes: an analysis of 5,951 cases. Microsurgery 2017;37(8):858-64.

[38] Piazza C, Taglietti V, Nicolai P. Reconstructive options after total laryngectomy with subtotal or circumferential hypopharyngectomy and cervical esophagectomy. Curr Opin Otolaryngol Head Neck Surg 2012;20(2):77-88.

[39] Nouraei SA, Dias A, Kanona H, Vokes D, O'Flynn P, Clarke PM, et al. Impact of the method and success of pharyngeal reconstruction on the outcome of treating laryngeal and hypopharyngeal cancers with pharyngolaryngectomy: a national analysis. J Plast Reconstr Aesthet Surg 2017;70(5):628-38.

[40] Clark JR, Gilbert R, Irish J, Brown D, Neligan P, Gullane PJ. Morbidity after flap reconstruction of hypopharyngeal defects. Laryngoscope 2006;116(2):173-81.

[41] Godballe C, Jorgensen K, Hansen O, Bastholt L. Hypopharyngeal cancer: results of treatment based on radiation therapy and salvage surgery. Laryngoscope 2002;112(5):834-8.

[42] Pingree TF, Davis RK, Reichman O, Derrick L. Treatment of hypopharyngeal carcinoma: a 10-year review of 1,362 cases. Laryngoscope 1987;97(8 Pt 1):901-4.

[43] Kim S, Wu HG, Heo DS, Kim KH, Sung MW, Park CI. Advanced hypopharyngeal carcinoma treatment results according to treatment modalities. Head Neck 2001;23(9):713-7.

[44] Mahieu R, Colletti G, Bonomo P, Parrinello G, Iavarone A, Dolivet G, et al. Head and neck reconstruction with pedicled flaps in the free flap era. Acta Otorhinolaryngologica Italica 2016;36(6):459-68. 